\title{
Optical bedside monitoring of cerebral perfusion: technological and methodological advances applied in a study on acute ischemic stroke
}

\author{
Oliver Steinkellner \\ Physikalisch-Technische Bundesanstalt \\ Abbestr. 2-12 \\ 10587 Berlin, Germany
}

\section{Clemens Gruber}

Charité Universitätsmedizin Berlin

Neurologische Klinik

Charitéplatz 1

10098 Berlin, Germany

\section{Heidrun Wabnitz}

Alexander Jelzow

Physikalisch-Technische Bundesanstalt

Abbestr. 2-12

10587 Berlin, Germany

\section{Jens Steinbrink}

Jochen B. Fiebach

Charité Universitätsmedizin Berlin

Center for Stroke Research Berlin

Charitéplatz 1

10098 Berlin, Germany

\section{Rainer Macdonald}

Physikalisch-Technische Bundesanstalt

Abbestr. 2-12

10587 Berlin, Germany

\author{
Hellmuth Obrig \\ Charité Universitätsmedizin Berlin \\ Neurologische Klinik \\ Charitéplatz 1 \\ 10098 Berlin, Germany \\ and \\ Universitätsklinik Leipzig \\ Klinik für Kognitive Neurologie \\ Liebigstr. 16 \\ and \\ MPI für Kognitions und Neurowissenschaften \\ Stephanstr. 1a \\ 04103 Leipzig, Germany
}

\begin{abstract}
We present results of a clinical study on bedside perfusion monitoring of the human brain by optical bolus tracking. We measure the kinetics of the contrast agent indocyanine green using time-domain near-IR spectroscopy (tdNIRS) in 10 patients suffering from acute unilateral ischemic stroke. In all patients, a delay of the bolus over the affected when compared to the unaffected hemisphere is found (mean: $1.5 \mathrm{~s}$, range: $0.2 \mathrm{~s}$ to $5.2 \mathrm{~s}$ ). A portable time-domain near-IR reflectometer is optimized and approved for clinical studies. Data analysis based on statistical moments of time-of-flight distributions of diffusely reflected photons enables high sensitivity to intracerebral changes in bolus kinetics. Since the second centralized moment, variance, is preferentially sensitive to deep absorption changes, it provides a suitable representation of the cerebral signals relevant for perfusion monitoring in stroke. We show that variance-based bolus tracking is also less susceptible to motion artifacts, which often occur in severely affected patients. We present data that clearly manifest the applicability of the tdNIRS approach to assess cerebral perfusion in acute stroke patients at the bedside. This may be of high relevance to its introduction as a monitoring tool on stroke units. ๑ 2010 Society of Photo-Optical Instrumentation Engineers. [DOI: 10.1117/1.3505009]
\end{abstract}

Keywords: optical imaging; cerebral perfusion; near-infrared spectroscopy; timedomain measurements; indocyanine green; bolus tracking.

Paper 10061SSR received Feb. 5, 2010; revised manuscript received Aug. 16, 2010; accepted for publication Sep. 1, 2010; published online Nov. 19, 2010.

\section{Introduction}

Magnetic resonance imaging (MRI) can be considered the goldstandard for the diagnosis of acute stroke. Structural information is provided by T1- and T2-weighted imaging with high spatial resolution and hemorrhage can be reliably differentiated from ischemia. ${ }^{1}$ With respect to ischemic stroke, patho-

Address all correspondence to: Oliver Steinkellner, Physikalisch-Technische Bundesanstalt (PTB), Abbestr. 2-12, 10587 Berlin, Germany. Tel: 49-30-34817603; Fax: 49-30-3481-7505; E-mail: Oliver.Steinkellner@ptb.de. physiologically relevant information is additionally provided by the complementary strengths of diffusion- and perfusionweighted imaging (dwMRI and pwMRI). While dwMRI delineates the (irreversibly) damaged tissue, pwMRI is sensitive to the potentially larger area of hypoperfusion. The area of perfusiondiffusion mismatch has been termed "tissue at risk," providing an operational definition of the pathophysiological concept of the penumbra. This tissue at risk has been the prime target of acute therapeutic intervention, especially thrombolysis. In

1083-3668/2010/15(6)/061708/10/\$25.00 @ 2010 SPIE 
the past decade, diagnostics in acute ischemic stroke have thus greatly improved by pathophysiologically grounded MRI.

On the contrary, monitoring of ischemic stroke in the subacute phase of the disease is based largely on the same principles as general intensive-care medicine. Undoubtedly, this has been of great benefit for the outcome of patients. ${ }^{2}$ Stroke units have been shown to improve outcome in ischemic stroke, which is partly due to a much earlier diagnosis and treatment of primarily nonneurological complications. However, follow-up neuroimaging is usually warranted only in the case of a clinical deterioration of the patient, because MRI necessitates the transport of the patient to the radiology department, interferes with continuous clinical care/monitoring, and is demanding with respect to costs of personnel and device. These difficulties motivate the search for alternative bedside neuromonitoring methods, ideally easy to use and low in cost.

Optical imaging has been successfully advocated to have this potential. ${ }^{3-6}$ It has been shown to successfully provide an index of cerebral perfusion. ${ }^{7}$ Several recent papers have applied a dye-bolus-based approach to detect delays in perfusion in the diseased area much in analogy to the gadolinium-aided perfusion-weighted imaging ${ }^{8,9}$ with MRI. Indocyanine green (ICG) strongly absorbs light in the near-IR spectral region. A bolus is intravenously administered and its passage through the sampled brain tissue is detected by an optical probe array. So far, mainly sparse detector arrays have been used, aiming at the difference in perfusion between the diseased and nondiseased hemispheres. ${ }^{9-11}$ Principally, however, it is possible to cover the whole surface of the brain by using imaging devices, which have been used in optical topography studies. ${ }^{12}$ In Sec. 4, we show data obtained by an eight-channel setup, which may be a realistic compromise between ease of application in a clinical setting and an improved sensitivity to focal areas of hypoperfusion in some ischemic strokes.

A major problem with the continuous-wave (cw) near-IR spectroscopy (NIRS) method is the substantial contribution of superficial absorption changes to the signal measured. ${ }^{13,14}$ In functional stimulation experiments, the use of an adequate control condition can help to differentiate between the signal pertaining to brain activation and the extracerebral contamination by systemic hemodynamic changes. On the contrary, in perfusion assessment by ICG bolus tracking, the dye passage through the skin will influence the signal, even in the absence of hemodynamic changes. Thus, the separation of extracerebral and cortical signal contributions requires alternative strategies. Several approaches have been pursued to discriminate between cerebral and extracerebral bolus signals: (1) frequency-domain ${ }^{15,16}$ and (2) time-domain methods, and (3) multidistance measurements, also termed spatially resolved spectroscopy. ${ }^{17}$

In this paper, we focus on the time-domain NIRS (tdNIRS) approach and discuss its potential benefits. For such time-resolved approaches, several methods have been introduced to analyze the time-resolved diffuse reflectance, e.g., using time-dependent mean partial path lengths, ${ }^{18}$ applying the microscopic Lambert-Beer law, ${ }^{5}$ or considering changes in the intensity of various time windows or their ratios. ${ }^{3,19}$

Alternatively, the changes in the moments of the distribution of times of flight (DTOF) of photons ${ }^{11,20}$ were analyzed. Here, we concentrate on the second centralized moment, that is, the variance of the DTOF. It has been demonstrated that data analysis based on moments of the DTOF of the diffusely reflected photons considerably facilitates discrimination of the cortical signal from superficial contributions. We show that this also holds in a clinical study and that it additionally leads to a reduction of motion artifacts commonly caused by severely affected patients.

We integrate methodological and technological advances to the report on our clinical assessment of perfusion at the bedside. First, we describe the modifications of our instrument, data analysis of the DTOF as well as of the bolus kinetics, and the suppression of artifacts to then report on a study assessing perfusion in 10 acute stroke patients.

\section{Instrument and Methods}

\subsection{Apparatus}

We applied our modularly designed tdNIRS brain imager ${ }^{4}$ configured to monitor the kinetics of ICG boluses in stroke patients by recording time-resolved diffuse reflectance. Major modifications include (1) laser sources, (2) number of source-detector pairs recorded in parallel, and (3) suppression of fluorescence contamination. Moreover, the instrument was technically approved for use in clinical trials. The arrangement is sketched in Fig. 1(a). Diode laser modules (BHLP-700, Becker\&Hickl $\mathrm{GmbH}$, Germany) with an output power of about $10 \mathrm{~mW}$ and a repetition rate of $50 \mathrm{MHz}$ provided picosecond pulses at a wavelength of $785 \mathrm{~nm}$, which is close to the absorption peak of the contrast agent ICG. The laser modules are of monolithic design and temperature stabilized. They have a fast warm-up

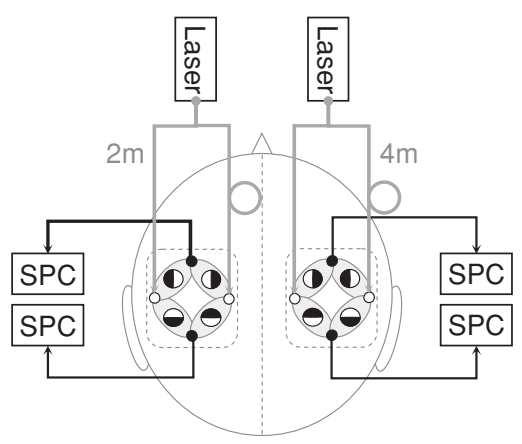

(a)
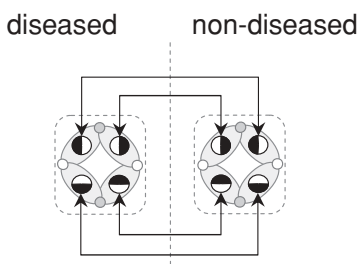

(b)

Fig. 1 Arrangement of optodes and their assignment to lasers and detection channels. (a) Laser light is coupled into two source fibers of different length. Together with four single-photon counting (SPC) channels, the arrangement enables us to measure at four different locations (optode pairs) over each hemisphere. (b) To assess perfusion delay between the diseased and nondiseased hemisphere, the corresponding locations are compared. The symbols encoding the respective positions apply also to Fig. 6. The affected side is always displayed on the left. 
time of about $10 \mathrm{~min}$ and a stable operating behavior, both essential for bedside application. Two modules, one for either hemisphere, were operated simultaneously. The laser output was equally split into two parts, which were coupled into multimode $600-\mu \mathrm{m}$-diameter glass fibers. Using different lengths of the fibers ( 2 and $4 \mathrm{~m})$, the pulses from the same source reached the light-emitting optodes with a temporal delay of $10 \mathrm{~ns}$. The bisection of the laser power represents a trade-off between laser power available and the number of source positions.

Due to the source splitting, two detection modules per hemisphere were sufficient to record the signals from four source-detector pairs on either hemisphere simultaneously [see Fig. 1(b)]. The detection modules contain photomultiplier tubes (R7400U-02, Hamamatsu Photonics, Japan), high-voltage power supplies, preamplifiers, and relay optics. In the presented absorption-based approach to track the bolus passage, fluorescence must be considered a signal contamination. Therefore, we inserted additional bandpass filters (L780-10, TFI Technologies, Inc., United States) into the optical path. This issue is discussed in more detail later in the paper. The four detection modules were connected to a multiboard time-correlated single-photon counting (TCSPC) system (SPC-134, Becker\&Hickl GmbH, Germany). The TCSPC boards associated with each hemisphere were triggered by the corresponding laser module. Histograms of the single-photon events representing DTOF from two sources delayed against each other were accumulated simultaneously in 1024 time channels of about 24.4 ps width and stored every $50 \mathrm{~ms}$. The count rates were adjusted between 2 and $3 \mathrm{MHz}$ for each detector. The instrument response function (IRF) was typically around $700 \mathrm{ps}$ (FWHM) wide, resulting from the laser pulse shape, temporal dispersion in the fiber bundles, and the transit-time spread in the photomultipliers.

For each hemisphere, two source fibers and two detection fiber bundles (diameter $4 \mathrm{~mm}$, length $2 \mathrm{~m}$; Loptek Glasfasertechnik, Germany) were mounted on an optode holder pad, in alternation on the vertices of a square with an edge length of $3 \mathrm{~cm}$. Since our focus was on infarctions in the middle cerebral artery (MCA) territory, the two identical pads comprising four measurement positions [Fig. 1(b)] were placed centrolaterally over positions $\mathrm{C} 3$ and $\mathrm{C} 4$ according to the 10-20 system. Hence, the area sampled included parts of the pre- and postcentral gyrus, partially reaching premotor areas and potentially parts of the inferior frontal gyrus. The pads and the headband were made with a biocompatible neoprene base. Medical grade elastic Velcro straps ensured a secure fixation on the patient's head. The arrangement and procedure were optimized for quick and secure mounting as well as patient comfort.

Generally, the setup allows for a modular increase in measurement positions. Note, however, that additional channels also increase the time to mount the device on the patient's head and lead to less flexibility of the approach, whose decisive strength is the bedside applicability. In our experience, the present tradeoff between channel number and technical demands as well as time to allow for a routine assessment is realistic in a clinical setting.

\subsection{Data Analysis}

The procedures described in the following are related to two different time scales of the measurement: (1) the nanosecond time scale $t$ of the DTOF, which we further term "micro time" and (2) the time scale $T$ concerning the passage of the contrast agent bolus in the range of seconds, denoted as "macro time".

Practically, analysis started with the processing of the DTOF on the micro time scale. After background subtraction, we calculated statistical moments $m_{k}$ of the histogram $N(t)$ of the diffusely reflected photons, ${ }^{20} m_{k}=\int_{t_{1}}^{t_{2}} t^{k} N(t) \mathrm{d} t$, where $k$ is the order of the moment. The total integral of the histogram $N_{\text {tot }}=m_{0}$, mean time of flight, i.e., the first normalized moment $\langle t\rangle=m_{1} / m_{0}$, and variance, i.e., the second centralized moment $V=m_{2} / m_{0}$ $-\left(m_{1} / m_{0}\right)^{2}$, were derived.

The use of moments features several advantages. First, for $\langle t\rangle$ and $V$, the contribution of the IRF can be simply eliminated by subtraction of the corresponding moment of the IRF. As a result, changes in the moments $\Delta\langle t\rangle, \Delta V$, as caused by a dye bolus, do not depend ${ }^{20}$ on the IRF. In contrast, analysis of the full DTOF or parts of it requires deconvolution procedures. Second, normalized moments do not depend on intensity and are therefore not affected by amplitude fluctuations. Third, variance does not depend even on the origin of the time axis. Hence, drifts in the temporal position of the DTOF do not influence the signal, as long as they are slow compared to the collection time intervals. These features of moments are relevant for the sensitivity to instrumental fluctuations as well as for the sensitivity to motion artifacts. Fourth, and not least, it was shown previously ${ }^{11}$ that variance is preferentially sensitive to deep absorption changes. Photons with large times of flight corresponding to long trajectories through the tissue enter with large weight $(t-\langle t\rangle)^{2}$. The probability that these photons provide information from the cortical layer is larger than for early photons. Therefore, variance allows for a reasonable discrimination of signal contributions originating from the cerebral cortex as opposed to changes in the skin and skull. Thus, changes in variance provide a reliable representation of the cerebral bolus passage, and we will focus on this signal in the analysis of patient measurements.

The integration limits $t_{1}$ and $t_{2}$ were fixed corresponding to fractions $\left(0.01\right.$ for $t_{1}$ and 0.03 for $\left.t_{2}\right)$ of the maximum photon count for a global DTOF averaged over the whole time series $(T)$. A proper choice of the upper integration limit is critical for the uncertainty of the moments calculated, in particular for variance. As stated, late photons are especially sensitive to absorption changes in the cortical layer of the volume sampled. Thus, overly reducing the integration range leads to considerable errors in the estimation of the cortical contribution. However, extending the integration range too far causes larger uncertainties in the moments due to background noise and hence increases the noise in the time courses. The influence of integration limits for moments was investigated previously in relation to the estimation of homogeneous optical properties. ${ }^{21}$ The choice of the integration limits used here was based on this work.

The macro time scale, which is of the order of seconds, is relevant for the extraction of the bolus kinetics from the time series of the recorded signals. In bolus tracking measurements, a fit of the gamma-variate function ${ }^{22}$ is widely used to analyze signal changes induced by the contrast agent. We applied this type of analysis to derive properties of the signals representing the passage of the boluses as a function of the macro time $T$. The gamma-variate function with a delay serves as the model 


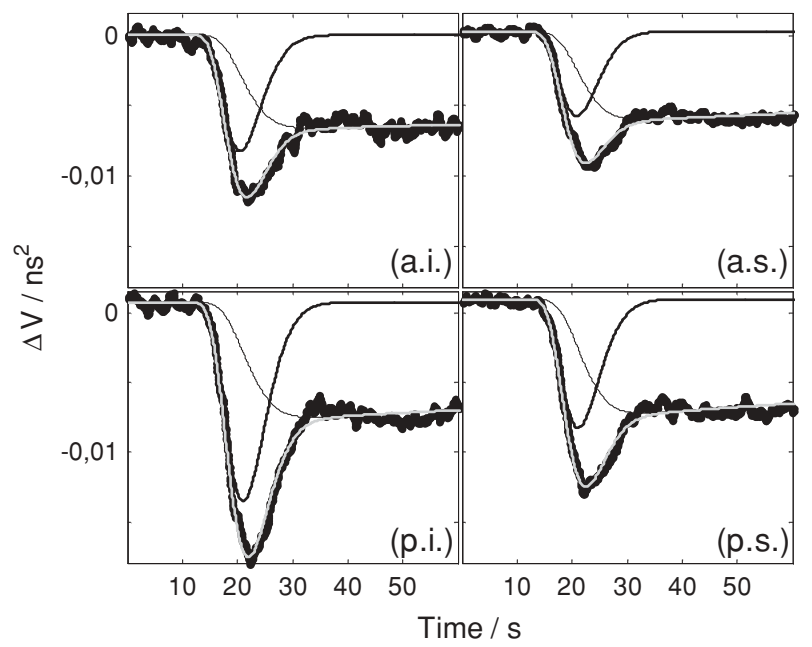

Fig. 2 Bolus passage recorded at four positions (a.i., anterior inferior; a.s., anterior superior; p.i., posterior inferior; and p.s., posterior superior) on the healthy hemisphere of a stroke patient. The black thick solid lines indicate the fitted gamma variate functions $C_{0}(T)$, and the black thin solid lines the cumulative integration over $C_{0}(T)$ with exponential decay. The gray solid lines show the overall fits $C(T)$.

function

$$
C_{0}(T)= \begin{cases}C_{p}\left(\frac{T-T_{0}}{T_{p}}\right)^{a} \exp \left(-a \frac{T-T_{0}}{T_{p}}\right) & \text { for } T \geq T_{0} \\ 0 & \text { for } T<T_{0}\end{cases}
$$

where the time to the bolus maximum $T_{p}$, the delay $T_{0}, C_{p}$ and $a$ are parameters to be determined by the fitting procedure. Since the function $C_{0}(T)$ approaches zero for short and long times $T$, a baseline must be subtracted prior to fitting Eq. (1) to experimental data. Time to peak, TTP $=T_{p}+T_{0}$, has proven to be a valid parameter to assess the perfusion also in case of an acute cerebral infarction. ${ }^{23}$ The peak value is $C_{0}(\mathrm{TTP})=C_{p} \exp (-a)$, and $a$ indicates the asymmetry of the curve.

To illustrate the fitting procedure, Fig. 2 shows the bolusinduced changes in all four source-detector pairs on the unaffected hemisphere of a stroke patient, based on the variance data. The signal drop due to the bolus passage in the cortex considerably differs among the positions. Beyond that, an additional signal contribution exists that decays on a much longer time scale. Since perfusion is slow in the extracerebral tissue, this component is assumed to result from the remaining superficial contribution and the gradual washout of the contrast agent. To account for the bolus passage in the extracerebral compartment, a cumulative integration over the bolus with an exponential decay is employed to fit this long-term behavior of the signal. Thus, the complete model function is

$$
C(T)=C_{0}(T)+b_{1} \int_{T_{0}}^{T} C_{0}\left(T^{\prime}\right) \mathrm{d} T^{\prime} \exp \left(-b_{2} \frac{T-T_{0}}{T_{p}}\right),
$$

where $b_{1}$ and $b_{2}$ are additional parameters determined by fitting. The full fits $C(T)$ (gray solid lines) were overlayed onto the measured curves. Note that TTP can be evaluated as minimum

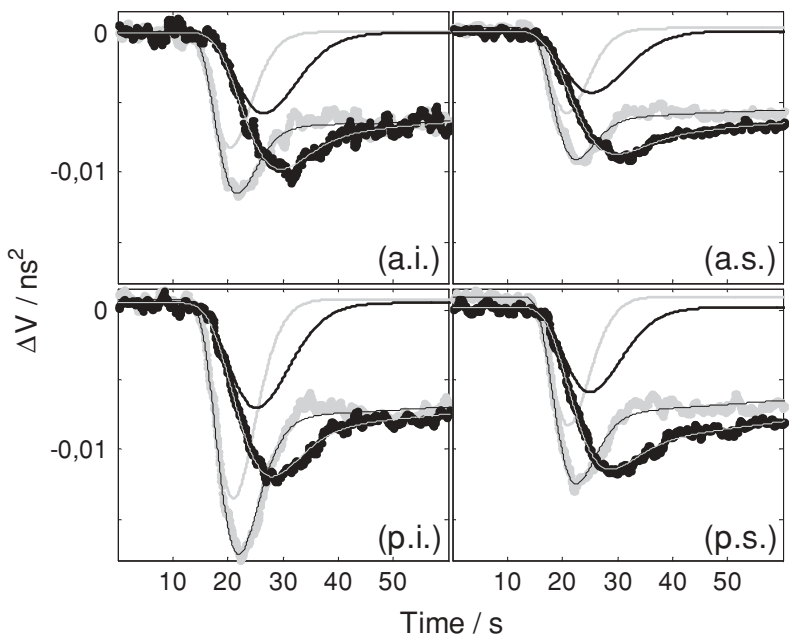

Fig. 3 Bolus passage recorded on four positions on the healthy (gray) and on the affected (black) hemisphere of the same stroke patient as shown in Fig. 2. The thick solid lines indicate the fitted gamma variate functions $C_{0}(T)$ and the thin solid lines show the overall fits $C(T)$. The bolus curves on the affected hemisphere are delayed and broadened with respect to the healthy hemisphere. The long-lived components appear very similar.

position of the bolus component $C_{0}(T)$ and of the full fit $C(T)$. The differences between these two approaches are discussed in the following.

To illustrate the influence of the fitting procedure on TTP, the target parameter used for perfusion monitoring in stroke patients, Fig. 3 shows the data of the affected (black lines) and the unaffected hemisphere (gray lines) in the same patient measurement. The corresponding components $C_{0}(T)$ of the fits (thick solid lines) again show a different time course than the full fits $C(T)$, which are overlayed onto the measured data. As expected, the bolus curves $C_{0}(T)$ recorded on the affected hemisphere are delayed and broadened when compared to the unaffected hemisphere. The long-lived components, however, appear very similar over both hemispheres. We conclude that cerebral perfusion has only minor influence on this component. On the other hand, the long-lived effect seen is a direct consequence of the bolus itself. In our simple model, we take this into account by the cumulative integration over $C_{0}(T)$. The exponential decay determining the signal shape on the scale of minutes is most likely due to the metabolism of ICG. It is slow compared to the bolus and does not influence the fit substantially.

To check the influence of eliminating the long-lived signal component, we determined TTP in two ways: (1) by evaluating the minima of the component $C_{0}(T)$ of the full fit $C(T)$ and (2) by direct estimation of the signal minimum (see Sec. 5). In the latter case, we used a truncated gamma-variate fit to determine the minimum of the original measured bolus curves.

To test the influence of the integration limits-on the micro time scale-on the analysis of the ICG bolus shape derived from integral and variance, we tentatively reduced both fractions defining $t_{1}$ and $t_{2}$ to 0.005 . On the macro time scale, the corresponding mean change in our target parameter TTP evaluated from all data sets and the corresponding standard deviation of the distribution (SD) were $0.05 \pm 0.50 \mathrm{~s}$ for the total integral and $0.06 \pm 0.27 \mathrm{~s}$ for variance. This shows that the integration limits, as used before, were chosen in a reasonable manner. 


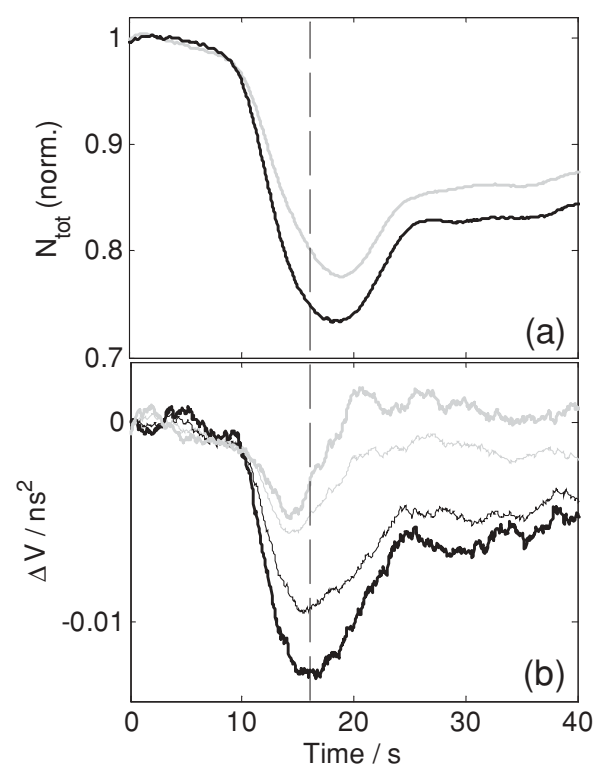

Fig. 4 Influence of fluorescence on the diffuse reflectance signals. The data show a bolus measured on a healthy volunteer at different positions, without (gray lines) and with (black lines) fluorescence blocking filter inserted for (a) normalized integral $N_{\text {tot }}$ and (b) change in variance $\Delta V$. Thick lines indicate data obtained for extended integration limits $(0.5 \%$, see text), thin lines in (b) for standard settings $(1 \%, 3 \%)$.

Note that in contrast to pwMRI measurements, the arterial input function, i.e., the time course of the bolus in the artery supplying the tissue under consideration, is currently not directly accessible by noninvasive optical measurements. Therefore, we focus on the analysis of differences in TTP between the affected and the unaffected hemispheres.

\section{Technical and Methodological Findings}

\subsection{Suppression of Fluorescence Contamination}

It has been demonstrated that laser-induced fluorescence of ICG circulating in the brain tissue can be detected and hence may be a candidate measure to assess brain pathology. ${ }^{24}$ However, in the approach of absorption-based bolus tracking, fluorescence must be regarded as a signal contamination. We checked the significance of this contamination and the efficiency of its suppression by a fluorescence-blocking filter by measurements on a healthy volunteer. Figure 4 compares the bolus shapes obtained with and without the fluorescence blocking filter. The time course of the integral trace [Fig. 4(a)] does not show a significant influence of the filter, while the amplitude difference can be attributed to the different measurement positions at which the same bolus was recorded. On the contrary, the time course of the variance signal [Fig. 4(b)] is substantially distorted by the fluorescence contribution. This differential influence of fluorescence on integraland variance-based bolus kinetics stems from the fact that - on the micro time scale - the maximum of the fluorescence signal appears considerably later than the peak of the reflected photons. Hence, fluorescence has a strong influence on the late photon counts, that is, the tail of the diffuse reflectance signal. Variance is rather susceptible to signal contaminations at late times because they enter with large weight $(t-\langle t\rangle)^{2}$. The integral signal is only marginally affected due to the low fluorescence quan- tum yield of ICG (3\% in physiological environment ${ }^{25}$ ), and the low ICG concentration. In particular, the position of absorption maximum is not altered.

Thus, the elimination of parasitic fluorescence is important in tdNIRS using variance-based perfusion measurements. To illustrate the distortions due to parasitic fluorescence, Fig. 4(b) shows the effects on a single bolus shape derived from measurements with (black lines) and without (gray lines) a fluorescence filter. On the macro time scale, fluorescence elicits (1) a substantial reduction of the magnitude of the bolus signal, (2) a shift of the minimum toward shorter times, and (3) an overshoot at the trailing edge of the bolus. Additionally, we tested the influence of the choice of the upper integration limit $t_{2}$ for variance. An extended interval (with $t_{2}$ at $0.5 \%$ of the maximum, thick solid lines) increases the distortions when compared to the usual value (with $t_{2}$ at $3 \%$ of the maximum, thin solid lines) since the shorter integration interval covers a much smaller part of the (late) fluorescence hump. With the blocking filter inserted (black curves) the position of the bolus minimum - on the macro time scaleno longer depends on $t_{2}$. Hence, to be sure that our results are not affected by fluorescence contamination we performed all patient measurements with fluorescence-blocking filters.

Though the relative insensitivity to parasitic fluorescence of the integral when compared to the variance-based measurements [Fig. 4(a) versus Fig. 4(b)] seems to advocate integral-based assessment, the figure also illustrates the crucial advantage of variance-based detection of the bolus passage. As can be clearly seen, the bolus peaks earlier when sensitivity to the deeper layers of the sampled volume is achieved. This conclusion rests on the fact that due to a much higher blood flow in the brain, the bolus passage through the cortex is expected to be earlier when compared to extracerebral tissue. ${ }^{11}$ This effect is relevant since variance-based, fluorescence-corrected bolus kinetics show a peak that is about $3 \mathrm{~s}$ earlier compared to the integral-based analysis.

\subsection{Reduction of Motion Artifacts}

Beyond such analytical considerations it is important to consider that in clinical measurements, especially in patients during the acute phase of a disabling disease such as stroke, movement artifacts can distort the assessment of the bolus shape. A severely affected patient may be unable to keep still even for a measurement period as short as several minutes. Therefore, we next discuss some aspects of the sensitivity to motion artifacts in our measurements.

The most prominent effects of motion are a change in optical coupling between optodes and skin or local variations in superficial optical properties, including changes in the amount of hair between skin and optodes. Such superficial changes will affect the amplitude of the DTOF only. Thus, the normalized moments should be less susceptible to such motion-related artifacts than the integral. In this study, we checked whether this prediction could be confirmed in our measurements on 10 patients suffering from acute stroke on whom we report in Sec. 4. Figure 5(a) displays the complete time course of a measurement including four bolus applications with a full duration of about $25 \mathrm{~min}$. Due to the severity of the infarction, the patient was not able to refrain from repetitive body and head movements during the measurements. The signals from two out of eight 


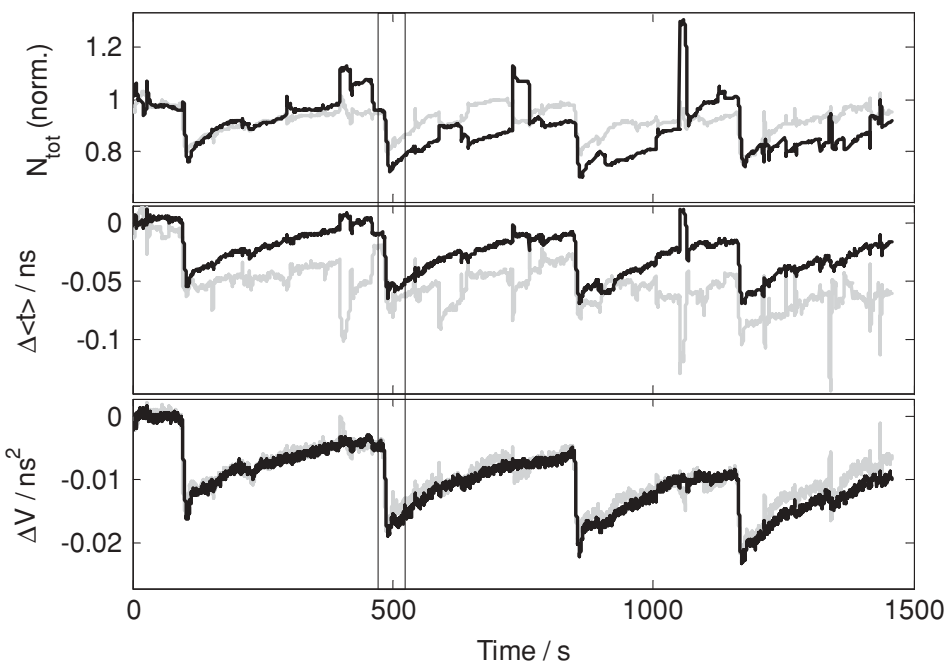

(a)

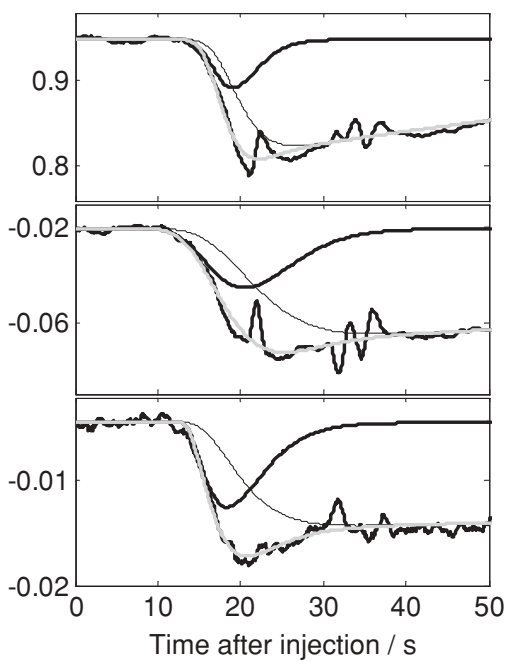

(b)

Fig. 5 Influence of motion: (a) time courses over the full length of a measurement in a patient (four boluses; $\sim 25$ min) and (b) zoom on a sequence of $50 \mathrm{~s}$ length from the gray curve at the second bolus. The area displayed is marked in (a). Fitted gamma-variate function $C_{0}(T)$ (black thick solid line), the cumulative integration over $C_{0}(T)$ (black thin solid line), and the overall fit $C(T)$ (gray solid line) are superimposed.

simultaneously recorded channels are shown. They were selected to illustrate different susceptibilities with respect to the patient's movements. The two positions correspond to the mediofrontal channels on either hemisphere. In the integral $N_{\text {tot }}$, the channel represented by the black curve shows strong artifacts almost as large as the changes induced by the ICG bolus. As expected, motion artifacts are dramatically reduced when mean time of flight $\langle t\rangle$ and even further when variance $V$ is used to assess bolus-induced changes.

A different behavior, however, is seen for the channel whose results are depicted in the gray curve of Fig. 5(a). While the signal changes assigned to motions of the patient show a minor influence on the time-integrated signal $N_{\text {tot }}$, motion artifacts gain importance on the first moment $\Delta\langle t\rangle$, whereas in the variance $\Delta V$ these motion-related signal contributions almost vanish again. In Fig. 5(b) a sequence of $50 \mathrm{~s}$ from this channel around the second bolus is magnified to show an example of the influence of motion artifacts on the fit of the bolus shape. A distinct motion artifact about $22 \mathrm{~s}$ after application of the contrast agent caused problems in the assignment of the signal minimum in the time-integrated signal $N_{\text {tot }}$ and to a greater extent at the first moment $\Delta\langle t\rangle$. On the trace of the variance $\Delta V$ these signal distortions virtually disappeared. Moreover, the disturbances visible between 30 and $40 \mathrm{~s}$ were largely reduced. In sum, based on these two types of susceptibilities to motion, the analysis of the variance parameter can be considered the best choice.

To determine whether this observation can be generalized, we inspected the whole set of measurements in 10 patients pertaining to the discrimination of motional artifacts and the signal contribution of the contrast agent. Signal changes induced by the contrast agent were compared to the changes elicited by the patient's motions. In about $60 \%$ of the recorded time courses, we observed a substantial reduction of motion artifacts for variance compared to the integral of the DTOF. In about $10 \%$, we noticed a deterioration of the signal. In all other cases, we saw only minor or no changes in the signal quality comparing the various moments. Note that variance is accessible in time-domain measurements only, while the integral reflects the result of a cw measurement. In summary, the improved immunity against motional artifacts is a direct consequence of the picosecond time resolution in conjunction with the moment analysis.

\section{Clinical Implementation}

Our preceding considerations resulted in (1) the application of the variance (micro time scale) using fluorescence suppression and (2) a fitting procedure using the full fit $C(T)$, Eqs. (1) and (2). These methods are used shortly in the evaluation of our clinical study. At the end, we compare the perfusion indices in this clinical sample when relying on such a variance based approach (only feasible by tdNIRS) to those that would be available to a cw-based approach.

\subsection{Bolus Procedure}

The contrast agent used, ICG (ICG Pulsion ${ }^{\circledR}$, Pulsion Medical Systems AG, Germany) is safe and frequently used for angiography in ophthalmology and, due to its rapid hepatic metabolization, is also applied to test liver function. The doses used in the presented approach are comparatively small: even half hourly application for pseudocontinuous stroke monitoring would not reach the maximal daily dose recommended by the safety guidelines. ${ }^{26}$ A daily maximum of $5 \mathrm{mg} / \mathrm{kg}$ body weight would equal $350 \mathrm{mg} /$ day in a patient of $70 \mathrm{~kg}$ weight. In the presented study, we applied several boluses of $6 \mathrm{mg}$ ICG diluted in $6 \mathrm{ml}$ of aqua injected manually into the cubital vein. After the bolus, the line was flushed with $20 \mathrm{ml}$ of saline. Up to four dye injections at a repetition time of about 5 min were performed in each patient. The whole procedure did not exceed a duration of half an hour.

\subsection{Patients}

Ten patients were recruited at two Stroke Units of the Department of Neurology, Charité, from August 2008 to March 2009. Patients were included when they presented with a neurologic 
Table 1 Clinical patient data.

\begin{tabular}{|c|c|c|c|c|c|c|c|c|}
\hline Patient & Gender & Age & Territory & Side & Etiology & TSO & pwMRI & NIHSS \\
\hline 1 & $\mathrm{~m}$ & 72 & MCA & L & ICA-Occl. & $\mathrm{TIAs} *$ & $n / a$ & 3 \\
\hline 2 & $f$ & 31 & $\mathrm{ACA} / \mathrm{MCA}$ & L & ICA-St, $M^{*}$ & $>1$ week & + & 2 \\
\hline 3 & $\mathrm{~m}$ & 81 & MCA & L & ICA-St, L $>>R$ & $1 d$ & + & 0 \\
\hline 4 & $f$ & 67 & MCA & $R$ & CE, Ascl & $1 d$ & + & 16 \\
\hline 5 & $\mathrm{~m}$ & 67 & MCA & $R$ & $\mathrm{CE}, \mathrm{Ascl}$ & $1 d$ & + & 5 \\
\hline 6 & $f$ & 78 & MCA & L & MCA-St, Ascl & $1 d$ & + & 0 \\
\hline 7 & $f$ & 69 & MCA & L & ICA-St & $1 d$ & $1+1$ & 10 \\
\hline 8 & $\mathrm{~m}$ & 61 & MCA & $R$ & Ascl & $1 d$ & + & 3 \\
\hline 9 & $\mathrm{~m}$ & 81 & $M C A$ & $R$ & ICA-St & $2 d$ & + & 3 \\
\hline 10 & $f$ & 64 & MCA & $R$ & CE, Ascl & $1 d$ & + & 5 \\
\hline Mean & $5 / 5$ & $67 \pm 14$ & & $5 / 5$ & & $\sim 1 \mathrm{~d}$ & & $5 \pm 5$ \\
\hline
\end{tabular}

Abbreviations: $m$, male; $f$, female; MCA, middle cerebral artery; ACA, anterior cerebral artery; $L$, left hemispheric lesion; $R$, right hemispheric lesion; ICA-St, stenosis of internal carotid artery; MCA-St, stenosis of MCA; Ascl, arteriosclerotic changes in extracranial/transcranial Doppler sonography; CE, cardiogenic embolus; ICA-Occl, occlusion of ICA; MM* , suspected Moya-Moya disease; TSO, time since onset of symptoms; TIAs*, transitoric ischemic attacks after a previous desobliteration of the right ICA and an acute occlusion of the left ICA.

In patients 1 and 2, a hemodynamic etiology is thus most probable.

deficit, compatible with a stroke in the MCA territory of either hemisphere. The patients included in the study presented due to an acute hemiparesis and/or mild to moderate aphasia, while the initial neurological status revealed additional signs [National Institutes of Health Stroke Scale (NIHSS) scores see Table 1]. Patients with severe aphasias and other deficits interfering with the mandatory consent procedure were excluded. Moreover, patients in whom the infarcted area was suspected or radiologically proven to not include any parts of the MCA territory were not enrolled in the study. In all patients, an MRI was performed on admission, which was within the first $24 \mathrm{~h}$ after symptom onset. The examination included dwMRI and in all but one patient (patient 1) also pwMRI. Patients gave informed consent to the study prior to inclusion and the protocol and procedures were approved by the local Ethical Committee.

Table 1 provides the clinical and epidemiological data for the patients. The side of the presenting stroke was balanced across subjects. In eight of the nine patients in whom pwMRI was performed, a clear increase in mean transit time, evidencing a perfusion deficit was seen in the affected hemisphere. Both optical perfusion assessment and MRI were performed within $12 \mathrm{~h}$ after hospital admission, which also meant that the patients were examined within 1 day after symptom onset. With respect to the etiology in patients 3 to 10 either arterial or cardiogenic thromboembolism was suspected based on the cardiovascular risk stratification performed during hospitalization. In patient 1 , a hemodynamic cause of the ischemia was suspected. This patient had previously undergone ICA desobliteration on the opposite side. The vascular diagnostics revealed an ICA occlusion on the affected side and a crossflow over the anterior communicating artery (ACoA). Though no pwMRI was performed, the delay in perfusion of the affected side can be assumed based on these findings. In patient 2, a distal ICA stenosis with a Moya-Moyatype vascular pathology was diagnosed. In this young patient, the presenting symptoms were an intermittent deterioration of preexisting deficits projecting to the left MCA territory, pwMRI disclosed a hypoperfusion in the MCA and ACA (anterior cerebral artery) territory of the affected side. Thus, a hemodynamic watershed ischemia was assumed.

\section{Clinical Results and Discussion}

\subsection{Clinical Study}

Figure 6 shows the results obtained in 10 patients measured during the acute stage of an ischemic stroke (for clinical characteristics see Table 1 and Sec. 4.2). We calculated the interhemispheric differences of TTP, which were derived from the $\Delta V$ time courses with the procedure described in Sec. 2.2. The $\Delta \mathrm{TTP}$ was calculated for each corresponding optode pair and each of the up to four bolus administrations. In patients 1, 2, 3, 6, and 7 the left hemisphere was affected, in the other patients, the right hemisphere. Figure 6(a) illustrates the differences between hemispheres when averaging across all channels and all boluses. The TTP difference affected-unaffected hemisphere in all cases reveals a delay in the bolus passage, which is in line with the assumption of an acute hypoperfusion of the affected hemisphere. Taking into account the averaged hemispheric differences, we found an overall delay on the affected hemisphere of $1.5 \pm 1.6 \mathrm{~s}$ and the range was 0.2 to $5.2 \mathrm{~s}$. Under the assumption that the perfusion deficit is distributed over the whole hemisphere we can use the standard error of mean (SEM), calculated from all differences of positions and all boluses in a patient to 

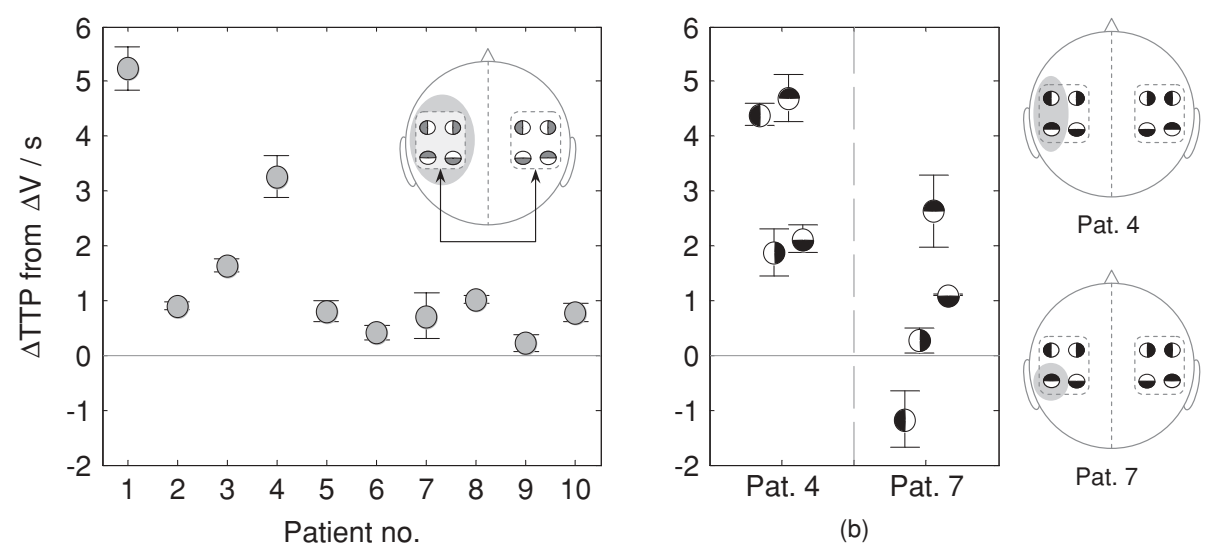

(a)

Fig. 6 Results of the perfusion measurements in 10 patients suffering from acute MCA(/ACA) stroke. (a) Mean delay between time-to-peak ( $\triangle T T P$ ) when subtracting the values of the nondiseased from the diseased hemisphere. Here, the delay was averaged across all measurement positions in each hemisphere (see inset). (b) In two patients, there was a clear difference between single channel comparisons (symbol coding as in Fig. 1). Patient 4 showed a much larger delay over the lateral channels, while patient 7 showed the largest delay in the posteriolateral optode pair.

characterize the reproducibility of our measurement. The SEM averaged over all patients was found to be $0.2 \mathrm{~s}$.

Though we did not quantitatively compare tdNIRS assessment with the pwMRI measurements performed in the acute stage in 9 of the 10 patients, qualitatively the data did reflect the results of the clinical and pwMRI-based assessment. In patient 1 an occlusion of the ipsilesional ICA was seen. Also the contralesional ICA had been desobliterated previously. Though in this patient no pwMRI was performed, a comparatively large delay of $5 \mathrm{~s}$ may be well explained by the long collateral route over the anterior communicating artery fed by the desobliterated contralesional ICA.

For the other patients, on average the delays were rather small compared to the delays reported in a similar study ${ }^{9}$ relying on cw NIRS technology and pwMRI. The latter study showed a mean delay of TTP in the NIRS measurements of around $3.6 \mathrm{~s}$, which is clearly higher than the here reported delays. Apart from differences in stroke severity (the average NIHSS reported in Ref. 9 was about 14, while it was 5 in this work), the large variability of TTP differences may partially be explained by the variability of the vascular pathology in the individual patient, notably preexisting ipsi- and contralateral stenosis of the large vessels. We do not focus on this variability here. However, with respect to our methodological focus in this paper, it is of interest that the study by Terborg et al. found a systematic overestimation of the TTP difference as assessed by NIRS when compared to the pwMRI assessment. ${ }^{9}$ On average, the delays estimated there in the pwMRI measurements were around $3 \mathrm{~s}$.

Another reason for the small delays observed in our study may result from the fact that the sampling volume covers only about $5 \mathrm{ml}$ within the upper cortical layer. Using pwMRI on territorial strokes, Neumann-Haefelin et al. ${ }^{27}$ showed that the ischemic core with perfusion delays of $8 \mathrm{~s}$ or more was located deep within the brain tissue. The surrounding tissue showed progressively less pronounced TTP delays. It is most likely that the sampled volumes did not reach the core of the ischemia in our patient measurements. They found that from the mean affected volume of about $134 \mathrm{ml}$, only $63 \pm 42 \%$ showed delays equal or larger than $2 \mathrm{~s}$. The delays found in our study are consistent with the assumption that our tdNIRS perfusion measurements were restricted to peripheral areas of the affected volume.

In patients $2,3,5,6,8,9$, and 10 the $\Delta$ TTP at the four different locations was quite similar, as is indicated by the small uncertainty of the mean value across measurements [error bars in Fig. 6(a), calculated as SEM]. In patients 4 and 7, on the contrary, the SEM was rather large. We therefore analyzed whether this variability was due to a difference between the bolus injections or due to a more focal hypoperfusion. Indeed patient 4 showed a clear difference between the more lateral locations (delay $>4 \mathrm{~s}$ ) and the medial locations (delay $<2 \mathrm{~s}$ ), as is sketched in the small upper insert of Fig. 6(b). This corresponded to the rather limited area of hypoperfusion seen in the pwMRI assessment. Similarly, in patient 7 , the largest delay was seen in the lateral posterior optode pair, as sketched in the lower inset of Fig. 6(b). Again this finding qualitatively corresponded to the rather focal hypoperfusion seen in the pwMRI, which was assessed a few hours prior to the optical measurement. Interestingly, the high interhemispheric delays are seen in these patients who had rather severe strokes (NIHSS of 14 and 10, see Table 1).

In sum, our data supply first evidence for the potential of our approach to have a coarse spatial differentiation of the hypoperfused area. This may be of relevance for a greater sensitivity to smaller ischemia and will also increase accuracy of the assessment in such instances.

\subsection{Discussion}

This section focuses on the effect of the signal-processing procedures onto the clinical results derived. In the following, results obtained using tdNIRS were compared to a representation of conventional $\mathrm{cw}$-technologies. Figure 7 summarizes the effect of different methods to determine TTP. The results obtained using our fitting procedure, as described in Sec. 2.2, are depicted by filled circles. Open circles show data, where the signal minimum has been identified using a least-squares fit of a gamma-variate function alone, truncated $5 \mathrm{~s}$ after the absolute signal minimum. This was applied only for the exact identification of the signal minimum. For this purpose, the use of the gamma-variate 


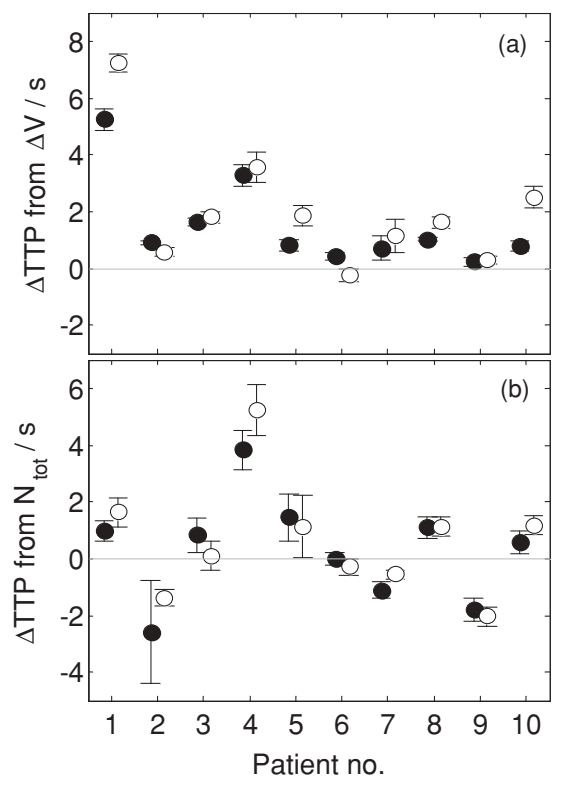

Fig. 7 Results of the perfusion measurements. The values of the nondiseased hemispheres were subtracted from the diseased ones and the delays were averaged across all measurement positions. The results obtained using the fitting routine are depicted in the filled circles, open circles show data from analysis of the signal minima without fitting the long lived component. Mean difference between $\Delta$ TTP values (a) when the variance $\Delta V$ was evaluated and (b) when the time-integrated signal $N_{\text {tot }}$ was evaluated.

function is not a prerequisite; basically, other suitable asymmetric functions could be employed. Note that Fig. 7(a) partially repeats data already shown in Fig. 6(a) for a better comparison. In 8 of 10 patients, the difference of affected and unaffected hemispheres is more pronounced for data obtained from the signal minimum without applying the (full) fit, however, also a much larger spread of the data was found. For the averaged hemispheric differences we found an overall delay on the affected hemisphere of $2.0 \pm 2.1 \mathrm{~s}$ and a range of -0.2 to $7.2 \mathrm{~s}$, when the signal minima were analyzed in such a way. As shown in the previous section, values derived by applying the full fitting procedure were $1.5 \pm 1.6 \mathrm{~s}$ and the range was 0.2 to $5.2 \mathrm{~s}$. The SEM averaged over all patients were 0.3 and $0.2 \mathrm{~s}$, respectively. We conclude that our fitting procedure leads to smaller but also more consolidated values than using the signal minimum alone.

To compare our tdNIRS approach relying on variance to the conventional cw-based approach only supplying the integral $\left(N_{\text {tot }}\right)$, we also calculated $\Delta$ TTP based on the time-integrated signal $N_{\text {tot }}$. The results are depicted in Fig. 7(b), which shows that in $40 \%$ of the patients, the bolus delay was found over the unaffected hemispheres (negative $\triangle \mathrm{TTP}$ in the figure). These findings are not compatible with the delays seen in the pwMRI assessment performed within hours of the optical measurements. Moreover, in the mean of all patients the $N_{\text {tot }}$ signal does not show a pronounced difference between the affected and the unaffected hemispheres at all. Without further fitting of the longlived component, the difference was $0.6 \pm 2.0 \mathrm{~s}$ with a range of -2.0 to $5.2 \mathrm{~s}$, applying the fit this difference was $0.3 \pm 1.8 \mathrm{~s}$ with a range of -2.6 to $3.8 \mathrm{~s}$. The SEM (averaged) found in this case were 0.7 and $0.6 \mathrm{~s}$, respectively.

\section{Conclusions}

We demonstrated that our tdNIRS approach is applicable in acute stroke patients to assess perfusion. In 10 patients, we showed that the expected perfusion delay of the diseased hemisphere could be seen in a TTP difference of the ICG bolus administered intravenously. The measurement configuration consisting of four source-detector pairs (channels) on either hemisphere is a reasonable trade-off between number of channels and technical as well as clinical demands. In single patients, a rough localization of the perfusion deficit within the diseased hemisphere was possible. Though localization of the perfusion deficit is clearly a domain of MRI-based perfusion imaging, a multichannel arrangement in our approach also improves the sensitivity to smaller cortical areas of hypoperfusion. Our results were qualitatively confirmed by pwMRI, which was performed in 9 of 10 patients. With regard to the clinical usefulness, note that the fixation of the optodes can be achieved within $10 \mathrm{~min}$. For the sake of a pseudocontinuous perfusion assessment the optode-holder pad can be optimized to remain on the patient's head, while only the optodes would have to be inserted prior to each measurement.

We previously showed that our time-domain approach is more suitable to record the truly intracerebral signal when compared to commercially available monitors, which are based on a cw approach. This is due to the depth sensitivity profile of the variance signal, which exhibits a maximum in a deeper region together with particularly low superficial sensitivity. In this work, we additionally showed that the time-domain approach also provides a substantial reduction of motion artifacts, which often occur in severely affected patients. This improvement is most distinct in the signals derived from variance of the DTOF. Note, however, that variance signals are particularly prone to contamination by a low, but nonnegligible contribution by ICG fluorescence. Insertion of a fluorescence-blocking filter easily solves this problem.

Our ongoing recruitment of patients will enable us to further explore the options for a longitudinal measurement, with the aim to complement initial pwMRI with a perspectively clinically relevant pseudocontinuous bedside monitoring of cerebral perfusion.

\section{Acknowledgments}

The research leading to these results received funding from the German Federal Ministry of Education and Research via the grants "Hypo- and Hyperfusion during subacute Stroke. Tracking Perfusion Dynamics in stroke Patients with Optical Imaging" (01GZ0710/01GZ0711) and "Center for Stroke Research Berlin" (01EO0801) as well as from the European Community's Seventh Framework Programme (FP7/2007-2013) under Grant Agreement No. FP7-HEALTH-2008-201076.

\section{References}

1. J. A. Chalela, C. S. Kidwell, L. M. Nentwich, M. Luby, J. A. Butman, A. M. Demchuk, M. D. Hill, N. Patronas, L. Latour, and S. Warach, "Magnetic resonance imaging and computed tomography in emergency assessment of patients with suspected acute stroke: a prospective comparison," Lancet 369, 293-298 (2007).

2. B. Fuentes and E. Diez-Tejedor, "Stroke units: many questions, some answers," Int. J. Stroke 4, 28-37 (2009). 
3. J. Selb, J. J. Stott, M. A. Franceschini, A. G. Sorenson, and D. A. Boas, "Improved sensitivity to cerebral hemodynamics during brain activation with a time-gated optical system: analytical model and experimental validation," J. Biomed. Opt. 10, 11013 (2005).

4. H. Wabnitz, M. Moeller, A. Liebert, A. Walter, R. Erdmann, O. Raitza, C. Drenckhahn, J. P. Dreier, H. Obrig, J. Steinbrink, and R. Macdonald, "A time-domain NIR brain imager applied in functional stimulation experiments," in Photon Migration and Diffuse-Light Imaging II, Proc. SPIE 5859, 58590H (2005).

5. D. Contini, A. Torricelli, A. Pifferi, L. Spinelli, F. Paglia, and R. Cubeddu, "Multi-channel time-resolved system for functional near infrared spectroscopy," Opt. Express 14, 5418-5432 (2006).

6. M. Kacprzak, A. Liebert, P. Sawosz, N. Zolek, and R. Maniewski, "Time-resolved optical imager for assessment of cerebral oxygenation," J. Biomed. Opt. 12, 034019 (2007).

7. I. G. Roberts, P. Fallon, F. J. Kirkham, P. M. Kirshbom, C. E. Cooper, M. J. Elliott, and A. D. Edwards, "Measurement of cerebral blood flow during cardiopulmonary bypass with near-infrared spectroscopy," $J$. Thorac. Cardiovasc. Surg. 115, 94-102 (1998).

8. C. Terborg, S. Bramer, S. Harscher, M. Simon, and O. W. Witte, "Bedside assessment of cerebral perfusion reductions in patients with acute ischaemic stroke by near-infrared spectroscopy and indocyanine green," J. Neurol. Neurosurg. Psychiatry 75, 38- 42 (2004).

9. C. Terborg, K. Gröschel, A. Petrovitch, T. Ringer, S. Schnaudigel, O. W. Witte, and A. Kastrup, "Noninvasive assessment of cerebral perfusion and oxygenation in acute ischemic stroke by near-infrared spectroscopy," Eur. Neurol. 62, 338-343 (2009).

10. E. Keller, A. Nadler, H. Alkadhi, S. S. Kollias, Y. Yonekawa, and P. Niederer, "Noninvasive measurement of regional cerebral blood flow and regional cerebral blood volume by near-infrared spectroscopy and indocyanine green dye dilution," Neuroimage 20, 828-839 (2003).

11. A. Liebert, H. Wabnitz, J. Steinbrink, M. Moeller, R. Macdonald, H. Rinneberg, A. Villringer, and H. Obrig, "Bed-side assessment of cerebral perfusion in stroke patients based on optical monitoring of a dye bolus by time-resolved diffuse reflectance," Neuroimage $\mathbf{2 4}$, 426-435 (2005).

12. M. A. Franceschini, D. K. Joseph, T. J. Huppert, S. G. Diamond, and D. A. Boas, "Diffuse optical imaging of the whole head," J. Biomed. Opt 11, 054007 (2006).

13. T. S. Leung, I. Tachtsidis, M. Tisdall, M. Smith, D. T. Delpy, and C. E. Elwell, "Theoretical investigation of measuring cerebral blood flow in the adult human head using bolus indocyanine green injection and near-infrared spectroscopy," Appl. Opt. 46, 1604-1614 (2007).

14. R. Mudra, A. Nadler, E. Keller, and P. Niederer, "Analysis of nearinfrared spectroscopy and indocyanine green dye dilution with Monte Carlo simulation of light propagation in the adult brain," J. Biomed. Opt. 11, 044009 (2006).

15. M. Kohl-Bareis, H. Obrig, K. Steinbrink, K. Malak, K. Uludag, and A. Villringer, "Noninvasive monitoring of cerebral blood flow by a dye bolus method: Separation of brain from skin and skull signals," $J$. Biomed. Opt. 7, 464-470 (2002).

16. J. Steinbrink, T. Fischer, H. Kuppe, R. Hetzer, K. Uludag, H. Obrig, and W. M. Kuebler, "Relevance of depth resolution for cerebral blood flow monitoring by near-infrared spectroscopic bolus tracking during cardiopulmonary bypass," J. Thorac. Cardiovasc. Surg. 132, 1172-1178 (2006).

17. H. W. Schytz, T. Wienecke, L. T. Jensen, J. Selb, D. A. Boas, and M. Ashina, "Changes in cerebral blood flow after acetazolamide: an experimental study comparing near-infrared spectroscopy and SPECT," Eur. J. Neurol. 16, 461-467 (2009).

18. J. Steinbrink, H. Wabnitz, H. Obrig, A. Villringer, and H. Rinneberg, "Determining changes in NIR absorption using a layered model of the human head," Phys. Med. Biol. 46, 879-896 (2001).

19. B. Montcel, R. Chabrier, and P. Poulet, "Detection of cortical activation with time-resolved diffuse optical methods," Appl. Opt. 44, 1942-1947 (2005).

20. A. Liebert, H. Wabnitz, J. Steinbrink, H. Obrig, M. Moeller, R. Macdonald, A. Villringer, and H. Rinneberg, "Time-resolved multidistance near-infrared spectroscopy of the adult head: intracerebral and extracerebral absorption changes from moments of distribution of times of flight of photons," Appl. Opt. 43, 3037-3047 (2004)

21. A. Liebert, H. Wabnitz, D. Grosenick, M. Moeller, R. Macdonald, and H. Rinneberg, "Evaluation of optical properties of highly scattering media by moments of distributions of times of flight of photons," Appl. Opt. 42, 5785-5792 (2003).

22. M. T. Madsen, "A simplified formulation of the gamma variate function," Phys. Med. Biol. 31, 1597-1600 (1992).

23. K. Yamada, O. Wu, R. G. Gonzalez, D. Bakker, L. Østergaard, W. A. Copen, R. M. Weisskoff, B. R. Rosen, K. Yagi, T. Nishimura, and A. G. Sorensen, "Magnetic resonance perfusion-weighted imaging of acute cerebral infarction: effect of the calculation methods and underlying vasculopathy," Stroke 33, 87-94 (2002).

24. A. Liebert, H. Wabnitz, H. Obrig, R. Erdmann, M. Moeller, R. Macdonald, H. Rinneberg, A. Villringer, and J. Steinbrink, "Non-invasive detection of fluorescence from exogenous chromophores in the adult human brain," Neuroimage 31, 600-408 (2006).

25. K. Licha, B. Riefke, V. Ntziachristos, A. Becker, B. Chance, and W. Semmler, "Hydrophilic cyanine dyes as contrast agents for nearinfrared tumor imaging: synthesis, photophysical properties and spectroscopic in vivo characterization," Photochem. Photobiol. 72, 392-398 (2000).

26. A. Raabe, J. Beck, R. Gerlach, M. Zimmermann, and V. Seifert, "Nearinfrared indocyanine green video angiography: a new method for intraoperative assessment of vascular flow," Neurosurgery 52, 132-139 (2003).

27. T. Neumann-Haefelin, H.-J. Wittsack, F. Wenserski, M. Siebler, R. J. Seitz, U. Mödder, and H.-J. Freund, "Diffusion- and perfusion-weighted MRI. The DWI/PWI mismatch region in acute stroke," Stroke 30, 15911597 (1999). 